\title{
Health Related Quality of Life among Osteoarthritis Patients: A Comparison of Traditional Non-Steroidal Anti-Inflammatory Drugs and Selective COX-2 Inhibitors in the United Arab Emirates Using the SF-36
}

\author{
Mohammed Hassanein*, Mohammed Shamssain, Nageeb Hassan \\ Clinical Pharmacy Department, College of Pharmacy and Health Science, Ajman University of Science and \\ Technology, Ajman, United Arab Emirates \\ Email: ${ }^{*}$ mohammedhassanein@outlook.com
}

Received 25 March 2015; accepted 27 April 2015; published 28 April 2015

Copyright (C) 2015 by authors and Scientific Research Publishing Inc.

This work is licensed under the Creative Commons Attribution International License (CC BY).

http://creativecommons.org/licenses/by/4.0/

(c) (i)

\section{Abstract}

Objectives: Osteoarthritis (OA) has a dramatic impact on patients' health related quality of life (HRQoL). Chronic use of analgesics and anti-inflammatory medications for pain management may improve symptoms but on long term may affect HRQoL negatively. The objective of the present study was to compare the impact of two different classes of analgesics, traditional non-steroidal anti-inflammatory drugs (NSAIDs) and selective cyclo-oxygenase-2 (COX-2) inhibitors on HRQoL among osteoarthritis patients using the SF-36 questionnaire. Methods: Clinic based cross-sectional study conducted at Al-Qassimi Hospital, Sharjah, United Arab Emirates (UAE), over a period of six months. Ethical Approval was obtained from the ethics committee at Al-Qassimi Clinical Research Center. Total of 200 osteoarthritis patients fulfilling the inclusion and exclusion criteria were involved in the study. Patients' demographics were collected from their medical records. The Medical Outcome Study Short-Form 36 (SF-36) questionnaire was used to measure patients' HRQoL. SF-36 data were scored using health outcomes scoring software 4.5. Results: Mean age of the subjects was $62.19 \pm 9.81$ years with females constituting $151(75.5 \%)$ of the patients. In general, females scored lower in most of the HRQoL domains compared to males and there was significant difference between the two groups in the mental health $(p=0.005) \&$ mental component $(p=0.042)$ domains. Compared to selective COX-2 inhibitors, patients on NSAIDs scored higher on all domains of SF-36 except physical functioning. There was significant difference in mental health

\footnotetext{
"Corresponding author.
} 
domain for patients treated with NSAIDs $(p=0.02)$. Celecoxib was only better than NSAIDs in osteoarthritis patients with more than one musculoskeletal disorders in the domain of bodily pain $(p=0.009)$. Conclusion: NSAIDs-treated patients did not differ significantly from celecoxib-treated patients in all domains of the SF-36 except for the mental health domain.

\title{
Keywords
}

\author{
Osteoarthritis, Health Related Quality of Life, Short Form-36, Traditional Nonsteroidal \\ Anti-Inflammatory Drugs, Selective COX-2 Inhibitors
}

\section{Introduction}

Osteoarthritis (OA) is a chronic disease that has a substantial effect on patients' health related quality of life (HRQoL) as well as an extremely high economic burden usually attributed to the treatment side effects [1]. Activities of daily living are negatively affected in OA patients [2]. Its symptoms are debilitating, and accounting for significant disability characterized as the impaired performance of social and physical life tasks [3] [4]. Their impact on patients' functional levels is well known and constitutes difficulties patients have to deal with, which will require long term pharmacological treatment and physical therapies [5].

The deleterious effects and impacts of osteoarthritis are well studied and mainly focusing on its consequences on health status; this is also true for the efficacy of treatments which was also assessed in context of health status and focused only on the objective measures of health status. However, few studies examined the impact of OA and its treatments on patients' HRQoL and the patients' subjective perspective. HRQoL outcomes provide an effective means for clinicians to make clinically sensible decisions by providing further insight into the benefits and drawbacks of treatment options [6] [7].

Pain and stiffness dominate other symptoms usually in early OA, therefore, treatment should focus on the reduction of pain and improvement of functional capacities as well as improvement of patients' HRQoL [8] [9]. Chronic use of analgesics and anti-inflammatory drugs can cause dramatic changes on patients' HRQoL \& long term plan is definitely important when safety and patients' HRQoL are considered. Previous studies have shown that both NSAIDs and selective COX-2 inhibitors improved patients' HRQoL when compared to the baseline [2] [3] [10] [11]; however very few studies conducted direct comparison between the two medication classes [12]. It is well recognized now that a key outcome measure for any intervention for OA is measuring the changes in HRQoL as for many other conditions [13].

According to the Emirates Arthritis Foundation (EAF), the estimated prevalence of arthritis in the UAE is $20 \%$, with only 6000 patients having been officially diagnosed [14]. The present study aims to compare the impact of two different classes of analgesics: traditional non-steroidal anti-inflammatory drugs (NSAIDs) and selective cyclo-oxygenase-2 (COX-2) inhibitors on HRQoL among osteoarthritis patients using the Medical Outcome Study SF-36 questionnaire as a measurement tool.

\section{Materials and Methods}

This was an observational cross-sectional study involving patients with symptomatic osteoarthritis recruited form Al-Qassimi Hospital in the United Arab Emirates. The present study was conducted between October 2013 and March 2014. Ethical Approval was obtained from the Ethics Committee of Al-Qassimi Clinical Research Center located at Al-Qassimi Hospital.

\subsection{Study Population}

Two hundred patients, 151 females and 49 males who fulfilled the inclusion and exclusion criteria were included in the study. To be eligible for inclusion, patients had to have documented diagnosis of osteoarthritis of any localization for at least 3 months, age $\geq 45$ years, taking either oral traditional NSAIDs or selective COX-2 inhibitors. Patients on topical NSAIDs, intra-articular hyaluronic injections or taking over the counter chondroitin or glucosamine supplementations were allowed to be involved in the study. Patients newly diagnosed; diagnosis 
dated back to less than 3 months, patients with concomitant oesteoarticular disorders, or with motor function impairment not due to osteoarthritis (e.g. Rheumatoid Arthritis), patients underwent knee replacement surgery, or on oral glucocorticoids were excluded from the study.

\subsection{Data Collection}

HRQoL was measured using the Medical Outcome Study Short Form-36 (SF-36) (4 weeks recall). The procedures for data collection were similar among the two groups. Each patient was identified by a code number for the purpose of maintaining patients' confidentiality and data retrieving. All subjects received an informed consent form asking them to participate in the study. Patients' socio-demographic data included gender, age, weight, height \& calculated Body Mass Index (BMI). Data regarding localization of OA, presence of any comorbidities or comorbid musculoskeletal disorders, concurrent OA medications, total daily doses of all the medications taken for osteoarthritis \& concurrent medications for other conditions were extracted from patients' files only after the questionnaire has been completed by the patient.

Comorbidities were classified into five categories; cardiovascular disorders including (hypertension, dyslipidaemia, ischemic heart diseases \& anaemia), endocrine and metabolic disorders including (diabetes mellitus both types, thyroid disorders), respiratory (asthma \& COPD), neuropsychiatric (stroke, epilepsy, Parkinson \& depression) and renal diseases. Obesity was regarded as one of the comorbidities and was defined by BMI.

\subsection{Measurement Tool}

The SF-36 [2] [3] [15] is a well-known self-administered and generic health status measure which encompasses 8 domains related to daily life activities and consist of 36 items. Each domain scores from 0 (lowest level of functioning) to 100 (highest level of functioning). Arabic version has been validated for the UAE Population. Both Arabic and English versions were available and readily distributed according to the patient's preferences. During the visit patients were invited to complete the whole questionnaire alone, only if required patients were assisted by a trained health professional or the principle investigator. All respondents were asked to answer the questionnaire based on what they understood.

\subsection{Statistical Analysis}

The data were analyzed using Statistical Package for Social Science (SPSS version 20). Descriptive analyses were used for socio-demographic data and clinical values. $p$ value $\geq 0.05$ was considered significant. Continuous variables were reported using means \& standard deviations (SD). Categorical variables were reported by percentage and proportions. SF-36 data was scored using the health outcomes scoring software 4.5. Chi-square test was used to examine the homogeneity of treatment groups for categorical variables. Independent sample t-test was used to assess the difference between traditional NSAIDs and selective COX-2 inhibitors in terms of HRQoL domains.

\section{Results}

Between October 2013 and March 2014, 200 osteoarthritis patients were eligible to be included in the study and agreed to complete the questionnaire. Written informed consent form was obtained from each patient. The English version of the SF-36 questionnaire was only used marginally by three patients (1.5\%). Reasons for non-participation were either patients were not interested or they did not have the time to complete the questionnaire.

Among the enrolled patients, 151 (75.5\%) were females and 49 (24.5\%) were males. The age range was between $45 \& 88$ years. Mean age was $62.19 \pm 9.81$. BMI was calculated using the reported weights and heights. Data on weights and heights was only available for 100 patients collectively. The other 100 patients were treated by SPSS as missing values. The definitions of the certain groups "normal", "overweight" and "obese" were based on the definition of the World Health Organization (WHO). Mean BMI was $32.68 \pm 8.82$ with females showed slightly higher values compared to males (Table 1 ).

NSAIDs were prescribed for $46.5 \%$ of the patients. Celecoxib was the only selective COX-2 inhibitors prescribed (53.5\%). With respect to the individual NSAIDs prescribed, meloxicam $7.5 \mathrm{mg}$ was the most common traditional NSAIDs prescribed among the study patients $(\mathrm{n}=81)(40.5 \%)$, followed by piroxicam $20 \mathrm{mg}(2.5 \%)$ 
Table 1. Physical measurements of subjects. (Mean \pm SD).

\begin{tabular}{cccc}
\hline Parameter & Females $(\mathbf{n}=\mathbf{1 5 1})$ & Males $(\mathbf{n}=\mathbf{4 9})$ & All \\
\hline Age (Years) & $60.77 \pm 9.312$ & $66.53 \pm 10.1$ & $62.19 \pm 9.817(\mathrm{n}=200)$ \\
Height (Cm) & $156.8 \pm 7.94$ & $167.8 \pm 10.0$ & $159.5 \pm 9.73(\mathrm{n}=100)$ \\
Weight (Kg) & $81.77 \pm 23.4$ & $89.58 \pm 31.69$ & $83.75 \pm 25.77(\mathrm{n}=100)$ \\
BMI & $33.0 \pm 8.66$ & $31.53 \pm 9.38$ & $32.68 \pm 8.82(\mathrm{n}=100)$ \\
\hline
\end{tabular}

\& diclofenac $50 \mathrm{mg}$ (2.5\%) with naproxen $200 \mathrm{mg}$ \& ibuprofen $400 \mathrm{mg}$ were marginally prescribed (0.5\%) for each, respectively (Table 2). Chi-square test did not reveal any significant difference between males and females in terms of the type of medications prescribed, but females were shown to be prescribed more analgesics both traditional NSAIDs \& selective COX-2 Inhibitors (celecoxib) more frequently compared to males.

In general, mental health domains and mental health component (mental health, role emotional, social functioning \& vitality) were better and less affected than physical health domains (physical functioning, role physical and bodily pain) for both genders. Females scored lower in all domains of SF-36 compared to males except for social functioning, however, the difference was not significant (Table 3). A significant difference was observed in the mental health domain between females and males $(p=0.005)$ who were already established on their medications (Traditional NSAIDs or COX-2 Inhibitors) at the time of the questionnaire administration as well as in the mental component summary $(p=0.042)$.

An independent t-test was performed to compare the HRQoL in patients taking traditional NSAIDs with those taking selective COX-2 inhibitors (Table 4). Patients on NSAIDs had higher scores of HRQoL than those on selective COX-2 inhibitors in all domains except for physical functioning domain but the difference was not significant. However, there was a significant difference in the domain of mental health in favour for NSAIDs over COX-2 inhibitors $(p=0.02)$. Meloxicam $7.5 \mathrm{mg}$ once daily was associated with better HRQoL in the domains of general health than celecoxib $200 \mathrm{mg}$ once daily $(p=0.053)$ and in the domain of mental health. Diclofenac was better than celecoxib in bodily pain domain $(p=0.025)$.

Patients with knee OA showed significant difference in the mental health domain between traditional NSAIDs group and selective COX-2 inhibitors $(p=0.047)$ in favour of NSAIDs. Patients with spine OA who were on traditional NSAIDs had better domains of SF-36 except for the vitality when compared with patients with knee OA. Significant difference was also found in favour for NSAIDs in patients with OA in more than one joint in the domains of role physical $(p=0.025)$ and bodily pain $(p=0.014)$ between NSAIDs and selective COX-2 Inhibitors respectively.

Patients with comorbidities scored lower in all domains of SF-36 compared to those without, with significant difference noticed in the role physical domain $(p=0.001)$. Presence of musculoskeletal comorbidity was highly correlated with increased bodily pain $(r=0.291)(p=0.018)$. Patients with other musculoskeletal disorders did not show to be different from those without, except when they were treated with celecoxib, there was a significant difference in the domain of bodily pain, where patient with other musculoskeletal disorders showed better HRQoL compared to those without $(p=0.009)$ (Table 5).

\section{Discussion}

The present study, to the best of our knowledge, is the first known study to examine the effects of different classes of analgesics associated with osteoarthritis on HRQoL of Arabic population. The use of the Medical Outcomes Study SF-36 questionnaire which was used in previous studies in patient with arthritis and it has been shown to has good responsiveness to changes in patients with rheumatic conditions compared with other longer instruments [16] [17], can be considered a strength of the present study. Unlike other instruments used to measure the functional impact of OA such as Western Ontario and MacMaster Universities Osteoarthritis (WOMAC), SF-36 is a multidimensional instrument and allow more global insight and assessment on different components of quality of life particularly in OA patients where comorbidities are common.

Females scored lower in all domains of SF-36 compared to males except for the domain of social functioning, where they showed to have similar quality of life. Many studies have shown that there are differences in HRQoL between the two genders [10] [15] [18] [19]. In general, mental health domains and mental health component 
Table 2. Frequencies of types of medications prescribed.

\begin{tabular}{|c|c|c|c|c|}
\hline \multirow[t]{2}{*}{ Types of Medications } & \multicolumn{2}{|c|}{ Frequency } & \multirow{2}{*}{$\begin{array}{c}\text { Total } \\
\text { Females \& Males }\end{array}$} & \multirow{2}{*}{$\begin{array}{c}\text { Percent } \\
(n=200)\end{array}$} \\
\hline & Females $(n=151)$ & Males $(n=49)$ & & \\
\hline \multirow[t]{2}{*}{ Traditional NSAID's } & $(n=69) 45 \%$ & $(n=24) 49.0 \%$ & 93 & $46.5 \%$ \\
\hline & $(\mathrm{n}=62) 31.0 \%$ & $(\mathrm{n}=19) 38.3 \%$ & 81 & $40.5 \%$ \\
\hline $\begin{array}{l}\text { Meloxicam } 7.5 \mathrm{mg} \\
\text { Diclofenac } 50 \mathrm{mg}\end{array}$ & $(\mathrm{n}=3) 1.5 \%$ & $(\mathrm{n}=2) 4.1 \%$ & 5 & $2.5 \%$ \\
\hline Naproxen $200 \mathrm{mg}$ & $(n=1) 0.7 \%$ & $(\mathrm{n}=0)$ & 1 & $0.5 \%$ \\
\hline \multirow{2}{*}{ Piroxicam 20 mg } & $(\mathrm{n}=1) 0.7 \%$ & $(\mathrm{n}=0)$ & 1 & $0.5 \%$ \\
\hline & $(\mathrm{n}=3) 2.0 \%$ & $(n=2) 4.1 \%$ & 5 & $2.5 \%$ \\
\hline Selective COX-2 Inhibitors & $(n=81) 40.5 \%$ & $(\mathrm{n}=26) 13.0 \%$ & 107 & $53.5 \%$ \\
\hline
\end{tabular}

Table 3. SF-36 domains and gender (Mean \pm SD).

\begin{tabular}{ccc}
\hline SF-36 Scores & Female $(\mathbf{n}=\mathbf{1 5 1})$ & Male $(\mathbf{n}=\mathbf{4 9})$ \\
\hline Physical Function & $43.36 \pm 20.23$ & $49.32 \pm 23.284$ \\
Role Physical & $40.14 \pm 26.36$ & $44.50 \pm 29.42$ \\
Bodily Pain & $36.54 \pm 16.26$ & $39.67 \pm 15.73$ \\
General Health & $52.04 \pm 17.80^{*}$ & $57.91 \pm 20.16$ \\
Vitality & $40.7 \pm 18.46^{*}$ & $49.47 \pm 22.21$ \\
Social Functioning & $68.79 \pm 27.11$ & $68.87 \pm 23.12$ \\
Role Emotional & $81.00 \pm 25.16$ & $82.99 \pm 23.87$ \\
Mental Health & $76.07 \pm 17.18^{* *}$ & $84.00 \pm 15.53$ \\
Physical Component & $34.62 \pm 10.90$ & $34.76 \pm 9.77$ \\
Mental Component & $52.50 \pm 9.83^{*}$ & $55.74 \pm 9.42$ \\
\hline
\end{tabular}

Note: SF-36 = Short Form-36, NS = Not significant $;{ }^{*} p \leq 0.05 ;{ }^{* *} p \leq 0.01 ; ; \stackrel{*}{* * *} \leq 0.001$.

Table 4. SF-36 Scores and types of medications prescribed (NSAID’s Vs. Coxibs). (Mean \pm SD).

\begin{tabular}{ccc}
\hline SF-36 Scores & Traditional NSAIDs $(\mathbf{n}=\mathbf{9 3})$ & Selective COX-2 Inhibitors $(\mathbf{n}=\mathbf{1 0 7})$ \\
\hline Physical Function & $43.65 \pm 20.49$ & $45.83 \pm 21.68$ \\
Role Physical & $43.80 \pm 27.90$ & $38.96 \pm 26.37$ \\
Bodily Pain & $39.19 \pm 17.33$ & $35.67 \pm 14.94$ \\
General Health & $55.75 \pm 18.27$ & $51.50 \pm 18.60$ \\
Vitality & $42.71 \pm 18.87$ & $42.96 \pm 20.57$ \\
Social Functioning & $69.75 \pm 26.15$ & $67.99 \pm 26.21$ \\
Role Emotional & $80.91 \pm 25.16$ & $75.70 \pm 27.06$ \\
Mental Health & $80.86 \pm 14.82 *$ & $75.54 \pm 18.57$ \\
Physical Component & $35.21 \pm 10.51$ & $34.16 \pm 10.72$ \\
Mental Component & $54.08 \pm 9.85$ & $52.62 \pm 9.76$
\end{tabular}

Note: SF-36 = Short Form-36, NS = Not significant; ${ }^{*} p \leq 0.05 ;{ }^{* *} p \leq 0.01 ;{ }^{* * *} p \leq 0.001$. 
Table 5. SF-36 domains and presence of comorbidities. (Mean \pm SD).

\begin{tabular}{|c|c|c|c|c|}
\hline SF-36 Domains & Presence of Comorbidity & Mean & $\mathbf{N}$ & Mean \\
\hline & \multicolumn{2}{|c|}{ Non-Musculoskeletal Disorders } & \multicolumn{2}{|c|}{ Musculoskeletal Disorders } \\
\hline \multirow{2}{*}{ Physical Function } & Yes (n = 135) & $44.24 \pm 21.60$ & Yes $(n=46)$ & $48.58 \pm 21.43$ \\
\hline & No $(n=65)$ & $46.02 \pm 20.17$ & No (n = 154) & $43.69 \pm 20.96$ \\
\hline \multirow{2}{*}{ Role Physical } & Yes $(n=135)$ & $36.75 \pm 26.11^{* * *}$ & Yes $(n=46)$ & $42.39 \pm 26.83$ \\
\hline & No $(n=65)$ & $50.48 \pm 27.04$ & No $(n=154)$ & $40.86 \pm 27.29$ \\
\hline \multirow{2}{*}{ Bodily Pain } & Yes $(\mathrm{n}=135)$ & $36.20 \pm 15.95$ & Yes $(n=46)$ & $41.73 \pm 14.55^{* * *}$ \\
\hline & No $(n=65)$ & $39.61 \pm 16.45$ & No $(n=154)$ & $35.98 \pm 16.41$ \\
\hline \multirow{2}{*}{ General Health } & Yes $(\mathrm{n}=135)$ & $51.40 \pm 18.73$ & Yes $(n=46)$ & $54.90 \pm 15.97$ \\
\hline & No $(n=65)$ & $57.80 \pm 17.44$ & No $(n=154)$ & $53.05 \pm 19.25$ \\
\hline \multirow{2}{*}{ Vitality } & Yes $(n=135)$ & $43.03 \pm 20.18$ & Yes $(n=46)$ & $45.38 \pm 19.15$ \\
\hline & No $(n=65)$ & $42.46 \pm 18.99$ & No $(n=154)$ & $42.09 \pm 19.92$ \\
\hline \multirow{2}{*}{ Social Functioning } & Yes $(\mathrm{n}=135)$ & $67.96 \pm 26.38$ & Yes $(n=46)$ & $72.82 \pm 24.33$ \\
\hline & No $(n=65)$ & $70.57 \pm 25.71$ & No $(n=154)$ & $67.61 \pm 26.60$ \\
\hline \multirow{2}{*}{ Role Emotional } & Yes $(n=135)$ & $78.20 \pm 26.31$ & Yes $(n=46)$ & $81.34 \pm 24.03$ \\
\hline & No $(n=65)$ & $77.94 \pm 26.37$ & No $(n=154)$ & $77.16 \pm 26.89$ \\
\hline \multirow{2}{*}{ Mental Health } & Yes $(n=135)$ & $77.55 \pm 17.65$ & Yes $(n=46)$ & $78.55 \pm 17.09$ \\
\hline & No $(n=65)$ & $78.98 \pm 15.99$ & No $(n=154)$ & $77.85 \pm 17.16$ \\
\hline \multirow{2}{*}{ Physical Component } & Yes $(n=135)$ & $34.17 \pm 11.86$ & Yes $(n=46)$ & $35.64 \pm 9.90$ \\
\hline & No $(n=65)$ & $35.65 \pm 7.35$ & No (n = 154) & $34.35 \pm 10.82$ \\
\hline \multirow{2}{*}{ Mental Component } & Yes $(n=135)$ & $52.99 \pm 9.99$ & Yes $(n=46)$ & $53.85 \pm 9.31$ \\
\hline & No $(\mathrm{n}=65)$ & $53.93 \pm 9.46$ & No $(n=154)$ & $53.13 \pm 9.97$ \\
\hline
\end{tabular}

Note: SF-36 = Short Form -36, NS = Not significant; ${ }^{*} p \leq 0.05 ;{ }^{* *} p \leq 0.01 ;{ }^{* * *} p \leq 0.001 ;:$ : Only with Celecoxib.

(mental health, role emotional, social functioning \& vitality) were better and less affected than physical health domains (physical functioning, role physical and bodily pain) for both genders, confirming the findings of the previous studies [10] [11].

We found significant difference between females and males in the domains of general health, mental health and Mental Component Summary. This can be explained by previous findings that emphasized on the biological and psychological differences between women and men [18]. This is can be of clinical importance since OA is more common in women than men. Our findings also suggest that females received analgesics more frequently than males, more specifically celecoxib. Previous conducted studies showed that there is a difference in prescribing patterns between the two genders, and they showed that women were significantly more likely to be prescribed an NSAID than men [6] [20].

Our results also suggest that compared to COX-2 inhibitors, NSAIDs treated patients scored higher on all domains of SF-36 except physical functioning, but the difference was not significant. However, we found significant difference between the two groups in the domain of mental health favoring NSAIDs. We found one study that conducted direct comparison between traditional NSAIDs and selective COX-2 inhibitors in terms of measuring HRQoL [12]. The study assessed the functional status and HRQoL of elderly osteoarthritis patients and showed improvement of HRQoL for both groups, on traditional NSAIDs naproxen and on celecoxib but could not find any significant difference between the two groups, confirming our results [12].

In terms of individual NASIDs, we found that meloxicam $7.5 \mathrm{mg}$ once daily, showed to have better effect 
when compared to celecoxib $200 \mathrm{mg}$ once daily in the domains of general health and mental health. We also found significant difference between diclofenac $50 \mathrm{mg}$ twice daily and celecoxib $200 \mathrm{mg}$ once daily in the domain of bodily pain where diclofenac had better effect than celecoxib. We found no difference between meloxicam $7.5 \mathrm{mg}$ once daily and the other NSAIDs used in the present study. This can be explained by the fact that meloxicam was the most traditional NSAIDs prescribed in our study and that other NSAIDs were marginally prescribed limiting our ability to detect any significant differences. That was the most common prescribing trend followed in the study setting despite the fact that meloxicam was shown to be marginally inferior to other traditional NSAIDs for providing pain relief \& for the symptomatic treatment of OA [21] [22]. Even though, we can attribute such trend in prescribing to the findings of different clinical trials that showed that meloxicam is associated with fewer endoscopic gastrointestinal ulcers and clinical and complicated upper gastrointestinal (UGI) events than non-selective NSAIDs, and thus more tolerated and accepted by the patients, although only the difference in clinical upper gastrointestinal events (peptic ulcer) reached statistical significance [21] [22].

With respect to the selective COX-2 inhibitors, celecoxib and etoricoxib are the only two medications in this class available in the UAE market. However, celecoxib was the only one available in the study hospital setting. We found that celecoxib was only better than NSAIDs in osteoarthritis patients with other comorbid musculoskeletal disorders. The difference was noticed only in the domain of bodily pain. This is probably can be explained by that such group of patients apparently required more analgesics and they were the most to benefit from their medications especially with an analgesic that is well tolerated by the patients . That was once reported in one of the studies but the effect was shown for NSAIDs [3], however, in that study, they did not include patients on selective COX-2 inhibitors which may explain the variances. Erich and co-workers reported that rofecoxib (COX-2 Inhibitors, withdrawn 2004 ) treatment increased physical and mental HRQoL domain scores on the SF-36 when compared with placebo and they claimed that the improvements in mental health with rofecoxib use primarily resulted from effective treatment of OA (i.e., reduction in pain and improvement in physical function) [11].

Our findings also suggest that females had more comorbidities compared to males, and they scored lower in all domains of HRQoL, especially the role physical domain where they showed significant difference. These results are independent of the fact that we have recruited more females than males and has been considered statistically. Studies focusing on comorbidity in OA patients showed that chronic conditions, such as hypertension, cardiovascular diseases, obesity, respiratory diseases and diabetes can be found alongside OA [23]. Previous studies showed that an increased prevalence of comorbidities was linked to a poorer HRQoL (SF-36 and WOMAC) [3] [6] [10]. On the other hand, one study showed no significant association between presence of comorbidity and HRQoL domains except for social functioning domain [6].

\section{Limitations}

A limitation of the present study that might has a potential impact on the results and that should be addressed in future studies is the rather heterogeneous sample of patients with regard to OA site; enrollment of a minority of patients with shoulder \& foot OA, thus limiting the generalizability of the results to these groups of patients. Future studies to investigate whether a specific site of OA would have any substantial effects on patients' HRQoL are encouraged.

\section{Conclusion}

Because there is no disease modifying treatments for osteoarthritis, reducing the pain severity and improving the HRQoL become the main goals of the disease management [16] [24]. Females were found to have poorer HRQoL compared to their male peers. Our findings suggest that NSAIDs-treated patients did not differ significantly from celecoxib-treated patients in all domains of the SF-36 except for the mental health domain. Mental health domains were shown to be better compared to physical health domains including physical functioning and bodily pain. Meloxicam $7.5 \mathrm{mg}$ once daily was associated with better HRQoL in the domains of general health and mental health compared to celecoxib.

\section{Acknowledgements}

The authors acknowledge the valuable support and collaboration of Dr. Assam Yahya, Senior Consultant Or- 
thopaedic Surgeon and the head of Joint and Reconstruction Unit at Al Qassimi Hospital and Dr. Dalia Moustafa Abo-Raya, Specialist Clinical Pharmacist, Deputy Head Drug Information Department at Al Qassimi Hospital in performing this study at Al-Qassimi Hospital—Sharjah—United Arab Emirates.

\section{References}

[1] Bryant, D. and Alldred, A. (2007) Rheumatoid Arthritis and Osteoarthritis. In: Walker, R. and Whittlesea, C., Eds., Clinical Pharmacy and Therapeutics, 4th Edition, Churchill Livingstone, China, 759-773.

[2] Majani, G., Giardini, A. and Scotti, A. (2005) Subjective Impact of Osteoarthritis Flare-Ups on Patients' Quality of Life. Health and Quality of Life Outcomes, 3, 14. http://dx.doi.org/10.1186/1477-7525-3-14

[3] Rabenda, V., Burlet, N., Ethgen, O., Raeman, F., Belaiche, J. and Reginster, J. (2005) A Naturalistic Study of the Determinants of Health Related Quality of Life Improvement in Osteoarthritic Patients Treated with Non-Specific NonSteroidal Anti-Inflammatory Drugs. Annals of the Rheumatic Diseases, 64, 688-693. http://dx.doi.org/10.1136/ard.2004.026658

[4] Geba, G., Weaver, A., Polis, A., Dixon, M., Schnitzer, T., et al. (2002) Efficacy of Rofecoxib, Celecoxib, and Acetaminophen in Osteoar Thritis of the Knee. JAMA: The Journal of the American Medical Association, 287, 64-71. http://dx.doi.org/10.1001/jama.287.1.64

[5] Breedveld, F. (2004) Osteoarthritis—The Impact of a Serious Disease. Rheumatology, 43, i4-i8. http://dx.doi.org/10.1093/rheumatology/keh102

[6] Zakaria, Z., Bakar, A., Hasmoni, H., Rani, F. and Kadir, S. (2009) Health-Related Quality of Life in Patients with Knee Osteoarthritis Attending Two Primary Care Clinics in Malaysia: A Cross-Sectional Study. Asia Pacific Family Medicine, 8, 10. http://dx.doi.org/10.1186/1447-056X-8-10

[7] Wiklund, I. (1999) Quality of Life in Arthritis Patients Using Nonsteroidal Anti-Inflammatory Drugs. Canadian Journal of Gastroenterology, 13, 129.

[8] Weiner, D. (2007) Office Management of Chronic Pain in the Elderly. The American Journal of Medicine, 120, 306315. http://dx.doi.org/10.1016/j.amjmed.2006.05.048

[9] Bijlsma, J., Berenbaum, F. and Lafeber, F. (2011) Osteoarthritis: An Update with Relevance for Clinical Practice. The Lancet, 377, 2115-2126. http://dx.doi.org/10.1016/S0140-6736(11)60243-2

[10] Briggs, A., Scott, E. and Steele, K. (1999) Impact of Osteoarthritis and Analgesic Treatment on Quality of Life of an Elderly Population. Annals of Pharmacotherapy, 33, 1154-1159. http://dx.doi.org/10.1345/aph.18411

[11] Dominick, K.L., Ahern, F.M., Gold, C.H. and Heller, D.A. (2004) Health-Related Quality of Life and Health Service Use among Older Adults with Osteoarthritis. Arthritis Care \& Research, 51, 326-331. http://dx.doi.org/10.1002/art.20390

[12] Lisse, J., Espinoza, L., Zhao, S., Dedhiya, S. and Osterhaus, J. (2001) Functional Status and Health-Related Quality of Life of Elderly Osteoarthritic Patients Treated with Celecoxib. The Journals of Gerontology: Series A: Biological Sciences and Medical Sciences, 56, M167-M175. http://dx.doi.org/10.1093/gerona/56.3.M167

[13] Brazier, J., Harper, R., Walters, S., Munro, J. and Sanith, M. (1999) Generic and Condition-Specific Outcome Measures for People with Osteoarthritis of the Knee. Rheumatology, 38, 870-877. http://dx.doi.org/10.1093/rheumatology/38.9.870

[14] Arthritis.ae. Emirates Arthritis Foundation (2013) http://www.arthritis.ae/index.php

[15] Rosemann, T., Laux, G. and Szecsenyi, J. (2007) Osteoarthritis: Quality of Life, Comorbidities, Medication and Health Service Utilization Assessed in a Large Sample of Primary Care Patients. Journal of Orthopaedic Surgery and Research, 2, 12. http://dx.doi.org/10.1186/1749-799X-2-12

[16] Bakas, T., Mclennon, S., Carpenter, J., Buelow, J., Otte, J., Hanna, K., Ellett, M., Hadler, K. and Welch, J. (2012) Systematic Review of Health-Related Quality of Life Models. Health and Quality of Life Outcomes, 10, 134. http://dx.doi.org/10.1186/1477-7525-10-134

[17] Angst, F., Aeschlimann, A., Steiner, W. and Stucki, G. (2001) Responsiveness of the WOMAC Osteoarthritis Index as Compared with the SF-36 in Patients with Osteoarthritis of the Legs Undergoing a Comprehensive Rehabilitation Intervention. Annals of the Rheumatic Diseases, 60, 834-840.

[18] Woo, J., Lau, E., Lee, P., Kwok, T., Lau, W., Chan, C., Chiu, P., Li, E., Sham, A. and Lam, D. (2004) Impact of Osteoarthritis on Quality of Life in a Hong Kong Chinese Population. The Journal of Rheumatology, 31, 2433-2438.

[19] Leveille, S., Zhang, Y., Mcmullen, W., Kelly-Hayes, M. and Felson, D. (2005) Sex Differences in Musculoskeletal Pain in Older Adults. Pain, 116, 332-338. http://dx.doi.org/10.1016/j.pain.2005.05.002

[20] Dominick, K.L., Ahern, F.M., Gold, C.H. and Heller, D. (2003) Gender Differences in NSAID Use among Older 
Adults with Osteoarthritis. Annals of Pharmacotherapy, 37, 1566-1571. http://dx.doi.org/10.1345/aph.1C418

[21] Chen, Y., Jobanputra, P., Barton, P., Bryan, S., Fry-Smith, A., Harris, G. and Taylor, R. (2008) Cyclooxygenase-2 Selective Non-Steroidal Anti-Inflammatory Drugs (Etodolac, Meloxicam, Celecoxib, Rofecoxib, Etoricoxib, Valdecoxib and Lumiracoxib) for Osteoarthritis and Rheumatoid Arthritis: A Systematic Review and Economic Evaluation. NIHR Evaluation, Trials and Studies Coordinating Centre (UK).

[22] Yocum, D., Fleischmann, R., Dalgin, P., Caldwell, J., Hall, D. and Roszko, P. (2000) Safety and Efficacy of Meloxicam in the Treatment of Osteoarthritis: A 12-Week, Double-Blind, Multiple-Dose, Placebo-Controlled Trial. JAMA Internal Medicine, 160, 2947-2954. http://dx.doi.org/10.1001/archinte.160.19.2947

[23] Van Dijk, G.M., Veenhof, C., Schellevis, F., Hulsmans, H., Bakker, J.P., Arwert, H., Dekker, J.H., Lankhorst, G.J. and Dekker, J. (2008) Comorbidity, Limitations in Activities and Pain in Patients with Osteoarthritis of the Hip or Knee. BMC Musculoskeletal Disorders, 9, 95. http://dx.doi.org/10.1186/1471-2474-9-95

[24] Centers for Disease Control and Prevention (2000) Measuring Healthy Days: Population Assessment of Health-Related Quality of Life. Centers for Disease Control and Prevention, Atlanta.

\section{List of Abbreviations}

BMI: Body Mass Index

COX-2: Cyclo-oxygenase 2

EAF: Emirates Arthritis Foundation

HRQoL: Health Related Quality of Life

NSAIDs: Nonsteroidal Anti-inflammatory Drugs

OA: Osteoarthritis

SF-36: Short Form-36

SPSS: Statistical Package for Social Science

SD: Standard Deviation.

UGI: Upper Gastrointestinal

UAE: United Arab Emirates

WHO: World Health Organization.

WOMAC: Western Ontario and MacMaster Universities Osteoarthritis 14 